\title{
Design Considerations on Feed-forward and Kalman Tracking Filters in Grid-Tied-Inverters Current-Control
}

\author{
João Cunha Ramos \\ INESC TEC and Faculty of Engineering \\ University of Porto \\ Porto, Portugal \\ joao.ramos@fe.up.pt
}

\author{
Rui Esteves Araújo \\ INESC TEC and Faculty of Engineering \\ University of Porto \\ Porto, Portugal \\ raraujo@fe.up.pt
}

\begin{abstract}
It is demonstrated that in grid-tied-inverter control, resonant integrators can be moved from the current loop to the input voltage filter allowing for better accuracy of voltage rms and frequency measurements whithout compromising controlability. Design of the input filter is discussed to ensure good performance and phase compensation and a robust feed-forward is built upon this filter that, together with the elimination of output distortion, results in a fair open-loop control response. By closing the loop with a PI controller and by turning off supposedly non-conducting IGBTs, a current transient results that competes with state-of-the-art control strategies and is only limited by output filter inductance while allowing for a fast and accurate estimator of grid parameters such as voltage rms and frequency, essential for fast droop control.
\end{abstract}

Keywords-Kalman filter, Frequency Locked Loop, Current control, Feed-forward, Dead-time distortion

\section{INTRODUCTION}

Standard approachs to current control in balanced threephase systems use Clarke and Park transforms and sequence estimation to achieve a reduced order DC representation in which simple PI control is easier to implement. Recently, the use of second order generalized integrators (SOGI) or linear kalman filters (LKF) has proven to be a more robust strategy for current control in strongly polluted networks [1] by processing voltages independently and even accounting for reduced order harmonics [2]. Using a frequency-locked-loop (FLL) based on this type of filter is also often discussed for measurement of AC frequency [3],[4]. In [5], a comprehensive analysis of the SOGI is undertaken and remarks are made over and its correct discretization and design.

This paper builds uppon this previous work, elaborating on how different aspects impact the performance of the linear kalman filter. Furthermore, the case will be made that moving the harmonic resonant integrators from the current loop to the kalman filter, increasing its number of states, is not only possible without compromising controllability as it also improves the accuracy of estimation of grid parameters as suggested in [6]. A voltage feed-forward is implemented based on the LKF and output distortion is reduced to improve controlability, accounting for all expected perturbances.
These perturbances include system delay, output impedance voltage drop and dead-time distortion. Dead-time distortion is compensated taking into account instantaneous current and the effect of current ripple in dead-time effective voltage, usually disregarded ([7],[8]). Since the design in consideration is for a micro-grid integrated converter, the stability of droopcontrol (either voltage or frequency) are also discussed and filter parameters are selected accordingly.

In section II, disturbances and feed-forward fundamentals are introduced and briefly discussed. Next, section III overviews the litterature on the Kalman filters and the impact of design choices in the filter accuracy and stability of droop-control mechanisms. Following, section IV presents the implementation of the feed-forward based on the Kalman and section $\mathrm{V}$ illustrates its effectiveness through simulation with MATLAB and Simulink.

\section{FEED-FORWARd IN CURRENT CONTROL}

For proper current control in a grid-tie inverter, the modulated output must match the line voltage plus the expected drops over the output filter. Defining current referece $I_{o}^{*}$, grid voltage $V_{g}$, estimates of filter resistance $\hat{R}_{f}$ and inductance $\hat{L_{f}}$, an open-loop control voltage output $V_{o}^{*}$ can be defined as

$$
V_{o}^{*}=V_{g}+\hat{R}_{f} I_{o}^{*}+\hat{L_{f}} \frac{d I_{o}^{*}}{d t}
$$

Given the delay from acquisition to output (at least one cycle, $T_{s}$ ), PWM distortion and mismatches in impedance estimate, the actual modulated voltage differs. Small errors can be canceled with proportional control, but as its gain is limited, it might not be suficient to eliminate steady-state error. This limitation is greater for reduced values of filter inductance.

$$
V_{L f}<L \frac{I_{o}-I_{o}^{*}}{T s} \Rightarrow K_{p}<L f_{s}
$$

Altough an integral component can complement proportional control to eliminate steady-state error, it increases current loop delay and, if not properly compensated, may resonate with a rapidly changing current reference. If the main disturbances are predictable and characterizable, they 


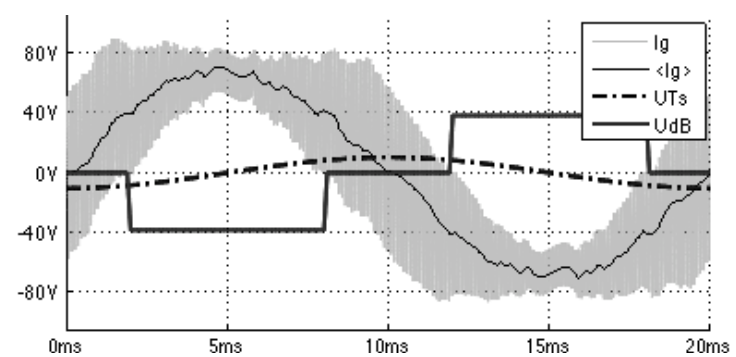

Figure 1. Cycle delay voltage and dead-band output distortion for $20 \mathrm{kHz}$ switching frequency and $3 \mu s$ dead-time. Cycle mean current and instantaneous current in the background scaled by $10 \mathrm{~V}: 1 \mathrm{~A}$.

can be taken into consideration, improving the open-loop response and eliminating the sole dependency on the closedloop response. A properly designed open-loop or feed-forward has faster convergence, is independent on error feedback and is more suited to highly dynamic systems.

The three main perturbances in current control of highfrequency converters are system delay, voltage drops and deadtime distortion. Delay of one sample time represents an error with magnitude $2 \pi f_{g} \sqrt{2} U_{r m s} T_{s} \approx 10 \mathrm{~V}$ at $10 \mathrm{kHz}$. Voltage drops can be up to $8 V$ at full load. Dead-time $(d T)$ distortion is given by $V_{d B}=-d T \cdot f_{s w} \cdot V_{d c} \cdot \operatorname{sign}\left(I_{o}\right)$ when both deadtime currents have the same sign (Figure 1).

As both impedance and dead-band distortion result in voltages against the direction of current, including them in the feed-forward results in natural negative feedback and control, as the effective proportional gain becomes $K_{p}+\hat{R}_{f}$ and deadtime intervenes with up to $\pm 40 \mathrm{~V}$ according to output current.

\section{KALMAN Filter BASEd CONTROL}

The use of first order integrators is not adequate for control and specially for filtering purposes in grid-tied applications given that, for a filter time constant $\tau_{i}$, results a group transport delay $t_{d}>\frac{\pi}{4} \tau_{i}$ which violates the strict synchronization restriction unless compensated. The use of second order generalized integrators (SOGI) or resonant integrators, with selfresonating frequency matching the grid, allows to eliminate this delay under steady-state conditions.

SOGIs have been used as current loop resonant integrators (see [1]) or as an input filter to extract the voltage fundamental, and even with frequency locked loop (see [3]). This approach eliminates the need for Clarke/Park transforms and sequence estimation, making the control lightweight and robust against voltage unbalance. Standard approach for harmonic elimination uses not one resonant integrator but one for each odd harmonic (up to 7th) in the current loop. These harmonic integrators can instead be incorporated in the Kalman filtering, improving its performance. A DC tracker (first order integrator) can also help to eliminate sensor bias. This will allow for complete elimination of tracking error in steady-state. The work in [2] is an example of such approach, although it should be noted that no quadrature correction is present and small angle approximation is used (see III-A and III-B). By tracking all harmonics, cross-effects are canceled and each component

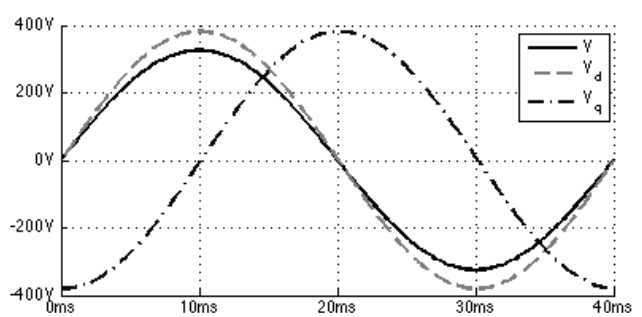

Figure 2. Effect of small angle approximation $\left(\tau_{U}=30 \mathrm{~ms}\right)$

has larger rejection ratio. Since the filter now reconstructs the input voltage with harmonics, it can be used as a feedforward with high-frequency noise rejection and even phase compensation (see IV-B).

It will be argued that through carefull design and by eliminating output distortion, it is possible to migrate the resonators from the current loop to the filter, making for better precision measurements of voltage and frequency without compromising controlability or performance.

\section{A. Second Order Generalized Integrator}

The operating principle of the SOGI is simple: given a stationary wave $V_{x}$, its derivative can be estimated from its integral since $s V_{x}=s^{2} \frac{1}{s} V_{x}$. If the integral is thus scaled and fed-back $\left(\frac{d V_{x}}{d t}=-\omega^{2} \int^{s} V_{x} d t\right)$ then the output resonates at the frequency $\omega$. If a tracking error is also fed-back then a system $\left[V_{d} ; V_{q}\right]$ of frequency $\omega$ results, $V_{d}$ in synchrony with the input and $V_{q}$ delayed $\frac{\pi}{2}$ or in quadrature. Along with no-delay filtering, this orthogonal system has interesting properties like constant amplitude measurement (3) and the ability for phase compensation through simple frame rotation.

$$
V_{r m s}=\frac{\left\|V_{d q}\right\|}{\sqrt{2}}=\sqrt{\frac{V_{d}^{2}+V_{q}^{2}}{2}}
$$

In [5], a comprehensive analysis of the SOGI is undertaken and remarks are made over filter design and its correct discretization. Most implementations, for simplicity reasons or pure mistake, use the small angle approximation for trigonometrics. Its impact on the SOGI's accuracy is hereby analysed, making the argument that it compromises accuracy.

1) Small Angle Approximation Sensibility Analysis: At every iteration, the proper evolution of the quadrature system is a rotation with a rotation matrix $A$ of angle $\theta=\omega T_{s}$

$$
\left[\begin{array}{l}
V_{d} \\
V_{q}
\end{array}\right]_{t}=\left[\begin{array}{cc}
\cos \left(\omega T_{s}\right) & -\sin \left(\omega T_{s}\right) \\
\sin \left(\omega T_{s}\right) & \cos \left(\omega T_{s}\right)
\end{array}\right]\left[\begin{array}{c}
V_{d} \\
V_{q}
\end{array}\right]_{t-T_{s}}
$$

Alternatively, an approximated matrix $A^{\prime}$ could be used:

$$
\left[\begin{array}{l}
V_{d} \\
V_{q}
\end{array}\right]_{t}=\left[\begin{array}{cc}
1 & -\omega T_{s} \\
\omega T_{s} & 1
\end{array}\right]\left[\begin{array}{l}
V_{d} \\
V_{q}
\end{array}\right]_{t-T_{s}}
$$

The resulting sine approximation error (6) results in a $8.2 \mathrm{mHz}$ out of $50 \mathrm{~Hz}$ deviation of the resonance frequency.

$$
\epsilon_{s}=\frac{\omega T_{s}}{\sin \left(\omega T_{s}\right)} \approx 1.00016451 \approx 165 p p m @ 10 k H z
$$


More pronounced is the non-unitary determinant (7) of this matrix, causing magnitude to increase nearly $0.1 \%$ every iteration. This is only limited by the filter's gain, resulting in large disturbance (8) for limited feedback gain (Figure 2).

$$
\begin{gathered}
\operatorname{det}\left(R^{\prime}\right)=1+\left(\omega T_{s}\right)^{2} \approx 1.00098696 \approx 100.1 \% \\
\epsilon_{o}=\frac{\operatorname{det}\left(R^{\prime}\right)-1}{K_{0} T_{s}}=\frac{\omega^{2} T_{s} \tau_{U}}{2} \approx 14.8 \%
\end{gathered}
$$

To avoid these errors, one can use a constant rotation matrix $A$ with fixed pre-calculated trigonometrics. However, for a frequency locked loop (FLL) to work, the matrix must be frequency dependent and so linearization near $f_{g}=50 \mathrm{~Hz}$ is best, given the small range of frequency of a grid-tie inverter, resulting in greater precision with equally light computation. A less precise alternative would be using $\sin \left(\omega_{n} T_{s}\right) \cdot \frac{f}{f_{n}}$ and fixed $\cos \left(\omega_{n} T_{s}\right)$.

\section{B. SOGI-based Kalman Filter}

Given the matrix A of state-space that reflects the natural response, a Kalman filter can be built simply by adding to expression (4) a correction component with the tracking error (9) and a Kalman gain vector as in (10). To fasten the convergence of the quadrature component, given its integrative nature by definition, the tracking error is let impact both $V_{d}$ and $V_{q}$ with quadrature gain as well as direct gain.

$$
\begin{gathered}
e_{x}(t)=V_{x}(t)-V_{d}(t-T) \\
{\left[\begin{array}{c}
V_{d} \\
V_{q}
\end{array}\right]_{t}=A\left[\begin{array}{l}
V_{d} \\
V_{q}
\end{array}\right]_{t-T_{s}}+\left[\begin{array}{c}
K_{d} \\
K_{q}
\end{array}\right] T_{s} \cdot e_{x}(t)}
\end{gathered}
$$

The gain can be determined from the desired time constant $\tau_{U}$ for the filter (instead of noise estimations) and a gain magnitude can be determined by (11). For best convergence, the quadrature correction gain has is optimum as (12) and the direct gain (13) results such that it preserves the above stipulated magnitude (see [5] for original expressions).

$$
\begin{gathered}
K_{0}=\frac{2}{\tau_{U}} \\
K_{q}=\omega\left[1-\sqrt{1+\frac{K_{0}}{\omega}}\right] \\
K_{d}=\sqrt{K_{0}^{2}-K_{q}^{2}}
\end{gathered}
$$

1) Amplitude Measurement and Droop-Control Stability: If the power reference is defined through a $\mathrm{P}(\mathrm{V})$ droop, it is crucial to avoid overshoot in the voltage amplitude estimate and related power oscilations, which happens for $K_{0}=2 \omega$ or $\tau_{U} \approx 3.2 \mathrm{~ms}$. If current-control and sensor delays are considerable, then the droop-power output should be low-passed with equivalent time constant. This results in $K_{q}=K_{0} \frac{1-\sqrt{3}}{2}$ and $K_{d}=K_{0} \sqrt[4]{3 / 4}$. In Figure 3 it can be seen that with $\tau_{U}=3.5 \mathrm{~ms}$ the amplitude falls with perceived $\tau_{U}=10 \mathrm{~ms}$. For values of $3.2 \mathrm{~ms}<\tau_{U}<10 \mathrm{~ms}$ the perceived time constant of the amplitude measurement does not decrease although tracking error reduces faster for $\tau_{U}=3.5 \mathrm{~ms}$.

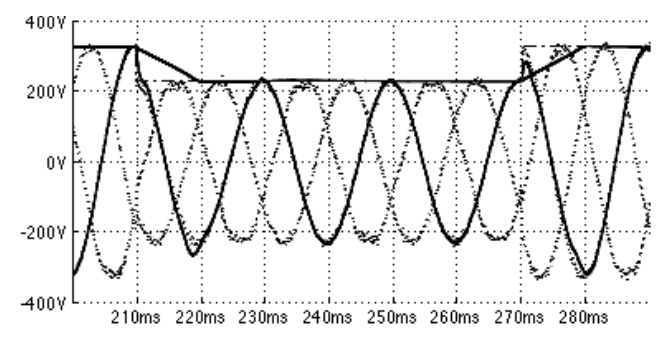

Figure 3. Tracking response to a $30 \%$ voltage sag $\left(\tau_{U}=3.5 \mathrm{~ms}\right)$. Voltage tracking of one phase and mean estimate of the three-phase amplitudes.

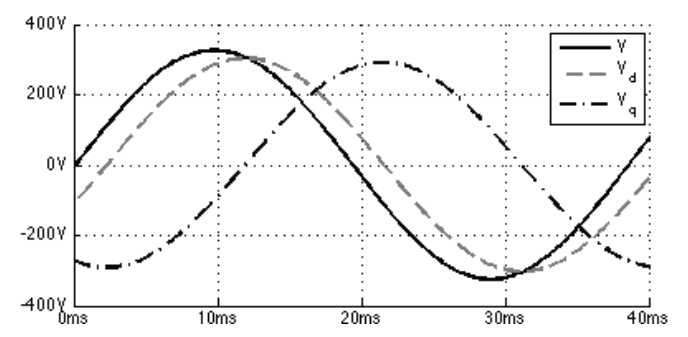

Figure 4. Delay of a $50 \mathrm{~Hz}$ SOGI without FLL tracking a $52 \mathrm{~Hz}$ input

\section{Frequency Locked Loop}

As with small angle approximation, a difference between the filter resonance frequency and the actual frequency will lead to tracking error (Figure 4). In [5], error is eliminated with variable sampling frequency of the algorithm. In [4], a FLL implementation is presented but the use of small error approximation and only one SOGI for the fundamental leads to oscilating behaviour in the frequency estimate in steady-state (from $\pm 200 \mathrm{mHz}$ to $\pm 1.8 \mathrm{~Hz}$ ). For fixed frequency PWM, the FLL is prefered, also resulting in a frequency measurement, facilitating the implementation of frequency power-droops, but requires careful implementation to achieve optimum results.

The FLL is achieved by feed-back of the voltage tracking error, multiplied by the quadrature component to give correct sign adjustments on frequency. The main causes of oscillation of the FLL in practical applications are sensor DC bias and the low order harmonics (as well as error from small angle approximation). By also tracking the DC and low harmonic voltage components (by moving the harmonic SOGIs from the current loop to the input filter) and increasing the number of states in the filter as suggested in [6], tracking is improved, error is canceled and frequency estimate becomes steady.

1) Frequency Measurement and Droop-Control Stability: As with the voltage droop, frequency estimate overshoot should be avoided. However, given the slower dynamics of frequency variation droop control based on frequency should be less susceptible to instability unless the network is isolated and has no synchronous generators or motors to ensure inertia. Still, the gain value for a critically dumped FLL, or to avoid overshoot and droop over-compensation, is deduced (Figure 5). For a frequency mismatch $\delta f$ results a tracking error (over a cycle $T_{s}$ ):

$$
\epsilon=\frac{\delta f}{f} \cdot \frac{d V_{x}}{d t} \cdot T_{s}
$$



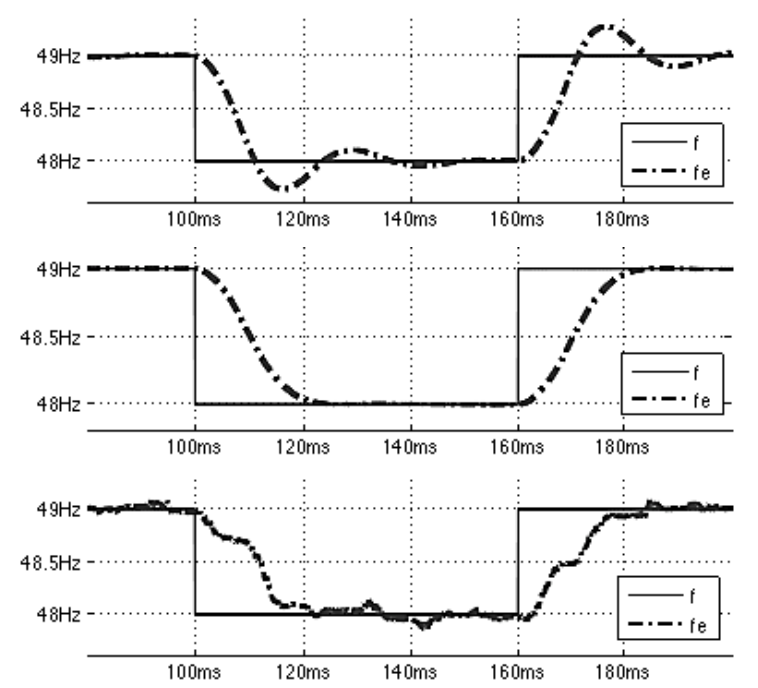

Figure 5. Response to a $1 \mathrm{~Hz}$ drop in frequency with $\tau_{f}=10 \mathrm{~ms}$ (top) and with no overshoot for $\tau_{f}=20 \mathrm{~ms}$ (middle) in a three-phase system. In single-phase, error gain is doubled to achieve a similar time constant (bottom)

Since the voltage tracking gain attenuates this error, the resulting contained error is $\delta V$ :

$$
\delta V=\frac{\epsilon}{K_{0} T_{s}}=\frac{\delta f}{f} \cdot \frac{d V_{x}}{d t} \cdot \frac{\tau_{v}}{2}
$$

Which can be expressed as a function of the quadrature $V_{q}$ since $\omega . V_{q}=-\frac{d V_{x}}{d t}$ or alternatively $\pi \cdot V_{q}=-\frac{1}{2 f} \frac{d V_{x}}{d t}$

$$
\delta V=-\delta f \cdot \pi V_{q} \cdot \tau_{v}
$$

Once tracking error is affected by the quadrature component, the expected time constant for the frequency locked loop for feedback gain $K_{f}$ becomes:

$$
\tau_{f}=\frac{\delta f}{K_{f} \cdot V_{q} \cdot \delta V}
$$

By depending on $V_{q}$, this means the convergence is faster at the flanks of the wave, due to larger $V_{q}$, also when the phase error translates to bigger voltage error ( $\delta V$ also proportional to $V_{q}$ ). However, in the three-phase system, by taking the three tracking errors together with $K_{f}\left(V_{a q} \delta V_{a}+V_{b q} \delta V_{b}+V_{c q} \delta V_{c}\right)$, the effects of the quadrature components add up to a constant feed-back and the response is smoother.

$$
V_{a q}^{2}+V_{b q}^{2}+V_{c q}^{2}=\frac{3}{2} V_{p k}^{2}
$$

And so, the frequency locked loop gain results:

$$
K_{f}=\frac{2}{\tau_{f} \cdot \tau_{v} \cdot 3 \pi V_{p k}^{2}}
$$

The critical value of $\tau_{f}$ is around the grid period $T_{g}=1 / f_{g}$, requiring that for no frequency estimate overshoot we have

$$
\tau_{f}>\frac{1}{f_{n}}
$$

For this value of $\tau_{f}=1 / f_{n}$ and also the critical value of the voltage tracking gain $\tau_{v}=1 / \omega_{n}$ that means

$$
K_{f}=\frac{4}{3}\left(\frac{f_{n}}{V_{p k}}\right)^{2}
$$

To avoid wind-up and instability, the frequency estimate is limited to a pre-defined range (e.g. $10 \%$ or $45-55 \mathrm{~Hz}$ ) with saturation. Also, to diminish the effects of sudden voltage sags during faults in the frequency estimate, one can limit its rate of transition. For a system of inertial constant $H$ (in seconds), and if the fault power or power imbalance is assumed below a certain maximum $\Delta P_{\max }$ the rate is limited to:

$$
\left|\frac{d f}{d t}\right|<\frac{\Delta P_{\max }}{P_{n}} \cdot \frac{f_{n}}{H}
$$

In single phase applications, the frequency is not corrected for $V_{q}=0$ making convergence less smooth and the gain should be doubled for the same time constant (Figure 5).

\section{Controller Implementation}

\section{A. Reference Generation}

The current reference vector is achieved through:

$$
\left[\begin{array}{c}
I_{\text {refd }} \\
I_{\text {ref } q}
\end{array}\right]=\frac{1}{V_{d}^{2}+V_{q}^{2}}\left[\begin{array}{cc}
P & Q \\
-Q & P
\end{array}\right]\left[\begin{array}{c}
V_{1 d} \\
V_{1 q}
\end{array}\right]
$$

As current reference is evaluated for each phase, current rises with voltage sags, maintaining equal power in all phases and constant current on the DC link, avoiding voltage ripple. If there is voltage ripple, however, the DC bus voltage controller should not agressively react to it or there will be an second harmonic in all three current reference vectors.

\section{B. Compensation of System Delay}

To avoid unnecessary delay, the analog conditioning circuit reject only very-high-frequency noise instead of performing a strong anti-aliasing which is instead achieved with synchronous sampling, aligning current measurements with the top and bottom of the PWM carrier (centers of the current ripple), and eliminate the ripple from the readings with a discrete FIR filter (average of the two double-frequency readings).

Then, at every control iteration, the voltage and current refer to present instantaneous measurements but the output to modulate refers to the next switching cycle and so there is a need to phase compensate or lead by $T_{s}$. Such compensation can be achieved simply by correcting the filter before frame rotation. This advances both grid voltages and current references by one sample time, accounting for control and PWM delays.

$$
\left[\begin{array}{c}
V_{d} \\
V_{q}
\end{array}\right]_{t}=A\left(\left[\begin{array}{c}
V_{d} \\
V_{q}
\end{array}\right]_{t-T_{s}}+\left[\begin{array}{c}
K_{d} \\
K_{q}
\end{array}\right] T_{s} \cdot e_{x}(t)\right)
$$

Additional phase lead can be performed, without compromising the filter, through additional frame rotation with $\theta=\omega t_{d}$ for all components of every phase, which might represent computational overhead, or alternatively, simply by adding a component $-\omega t_{d} V_{1 q}$ to the feed-forward (where $-\omega V_{1 q}$ approximates the voltage derivative). 


\section{Compensation of Voltage Drops}

The fixed $V_{c e 0}$ forward drop of the IGBT (if not MOSFET) should be subtracted from $V_{d c}$ when defining the output dutycycle (in (25) $V_{o s}$ is a modulation dependent offset, the positive zero sequence or the modulated neutral voltage).

$$
d=0.5+\frac{U_{o}+V_{o s}}{U_{d c}-U_{c e 0}}
$$

To compensate for total impedance (filter as well as transistor resistance $R_{e q}=R_{f}+2 \cdot R_{c e}$ and filter inductance $L_{f}$ ), a voltage drop component is generated from the current reference vector and added to the phase compensated voltage.

$$
V_{\text {drop }}=\left[\begin{array}{ll}
\hat{R}_{e q} & \omega T_{s} \hat{L}_{f}
\end{array}\right] \cdot\left[\begin{array}{c}
I_{r e f d} \\
I_{r e f q}
\end{array}\right]
$$

And thus results the output voltage feed-forward (27), reconstructed from all direct components and the impedance compensation, that should only require to be properly modulated (with no distortion) to ensure current control already thanks to the negative feed-back of impedance and dead-band distortion as mentioned earlier.

$$
V_{o}^{*}=\sum V_{i d}+V_{d r o p}
$$

\section{Compensation of Dead-time Distortion}

For this last perturbance to be properly compensated, the current and its ripple must be characterized within a switching cycle. In a single-phase topology with mixed PWM, current ripple amplitude (half of peak to peak) in an inverter leg can be estimated by

$$
I_{r}=\frac{\Delta I}{2}=t_{o n} \frac{V_{d c}-\left|V_{a}\right|}{2 L_{f}}=d_{o n} \frac{V_{d c}-\left|V_{a}\right|}{2 L_{f} f_{s}}
$$

If, in a switching cycle, the reference current is smaller than the ripple amplitude, it will cross zero and have opposing signs in the two dead-times resulting in no dead-time distortion and equation (25) holds. If on the other hand, $I_{o}^{*}>I_{r}$, then (29) is the dead-time compensated duty-cycle.

$$
d^{\prime}=0.5+\frac{U_{o}+o s}{U_{d c}-2 \cdot U_{c e 0}}+d T \cdot f_{s} \cdot \operatorname{sign}\left(I_{o}^{*}\right)
$$

With IGBT, since there is no reverse conduction, a better option would be to turn-on only the conducting transistor, eliminating dead-band altogether, halving gate losses and further improving transient response with free-wheeling.

In a three-phase converter, the ripple amplitude estimate depends on leakage and common-mode return currents. For significant capacitance of the common-mode return filter, neutral voltage can be considered constant within a cycle and:

$$
I_{r}=t_{o n} \frac{V_{d c}-\left(V_{a}+V_{o s}\right)}{2 L_{f}}
$$

For small or no capacitance, the inverter legs have crosseffect and ripple is reduced. It can however still be approximated by (30) with a reduction factor (tuned, depending on ammount of common-mode currents).

\section{E. Error feed-back and closed-loop control}

For each phase, the feed-forward is complemented with proportional control $\left(K_{p}<0.5 L_{f} f_{s}\right)$. Harmonic compensation is already performed by the voltage feed-forward but an additional SOGI at fundamental frequency is added to account for output impedance or delay mismatch.

\section{F. Output Impedance Estimation and Usage}

Due to temperature effects or differences in fabrication, filter impedance might vary, and the resulting mismatch to the stipulated value will be compensated by the feed-back loop and integrator (as can be seen in Figure 9, before adding feed-back, current drops slightly due to underestimation of resistance value).

An inverse of (26) can infer on these mismatches:

$$
\left[\begin{array}{c}
\Delta R \\
\omega T_{s} \Delta L
\end{array}\right]=\left(\frac{1}{I_{d}^{2}+I_{q}^{2}}\right)\left[\begin{array}{cc}
I_{d} & I_{q} \\
I_{q} & -I_{d}
\end{array}\right]\left[\begin{array}{c}
V_{1 d} \\
V_{1 q}
\end{array}\right]
$$

These error could be integrated into the impedance estimate migrating the integral control to the feed-forward but, for small mismatches, there is little added value. An estimate of the line impedance $Z_{g}$ (after voltage sensor) can also be considered to improve the response of both voltage droop an current control, by making the Kalman filter track $V_{g}-Z_{g} I_{o}^{*}$ instead of $V_{g}$ and including $Z_{g}$ in the $V_{d r o p}$ compensation. This way, voltage is controlled not at the end of the line but closer to the grid connection and point of common coupling.

\section{RESULTS}

In order to illustrate real-case scenarios, but without loss of generality, a $50 \mathrm{~Hz}$ frequency is assumed, as is a $1.75 \mathrm{mH}$ output filter inductance, $640 \mathrm{~V}$ bus voltage and $10 \mathrm{kHz}$ switching frequency with $3 \mu \mathrm{s}$ dead-time. Current of one leg out of a three-pole inverter is shown.

In Figure 6, the effects of dead-time distortion are visible, making it impossible for the current to detach from zero more than the ripple amplitude. In Figure 7, it is shown that proportional gain alone can only reduce current error to $1.4 \mathrm{~A}$ when current is greater than ripple. Integral component might redistribute the current error but has poor transient response and cannot ensure good response. In Figure 8 the effect of compensating dead-time distortion is almost equivalent to proportional control. By also adding compensation of output filter impedance voltage drop (Figure 9), current control is almost accurate, failing slightly due to filter heating and resistance underestimation. Finally by closing the loop with error feed-back, all remaining small signal error is corrected (Figure 10) with only slight error in the timing of dead-time compensation due to the approximation in ripple estimation, which effect all poles simultaneously and from common mode (zero-sequence) currents to the common-mode feed-back filter.

Last, Figure 11 illustrates a full scale transient which causes a voltage variation at the point of common coupling (PCC). Although the current corrects quickly, harmonic ellimination takes up to $20 \mathrm{~ms}$ as the Kalman filter tracks the new voltage. 


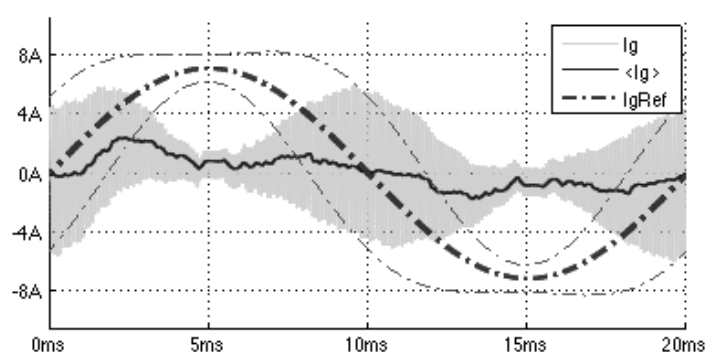

Figure 6. Open-loop current response with dead-time distortion

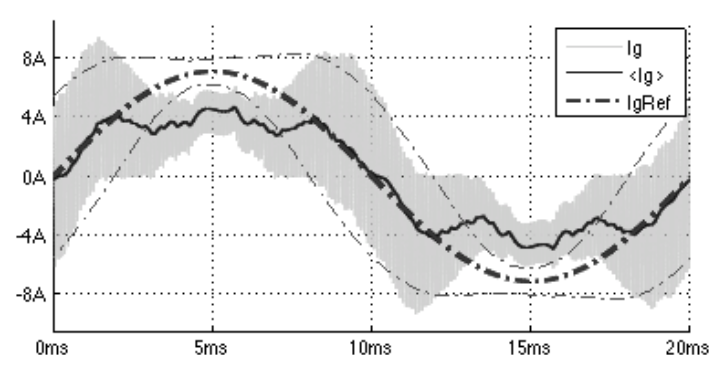

Figure 7. Closed-loop current response with dead-time distortion

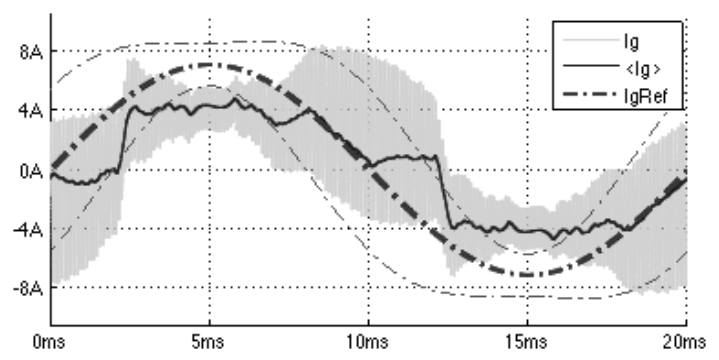

Figure 8. Open-loop current response with dead-time compensation only

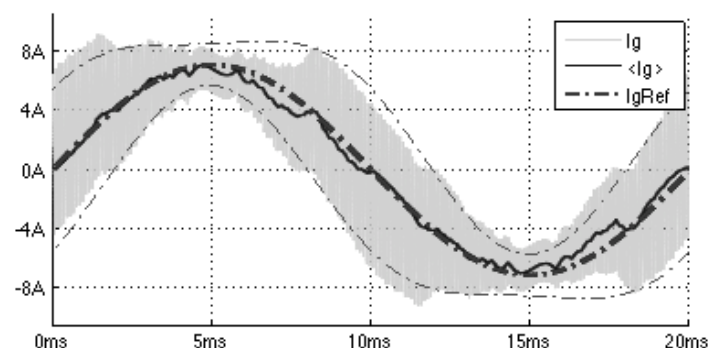

Figure 9. Open-loop with both dead-time and impedance compensations

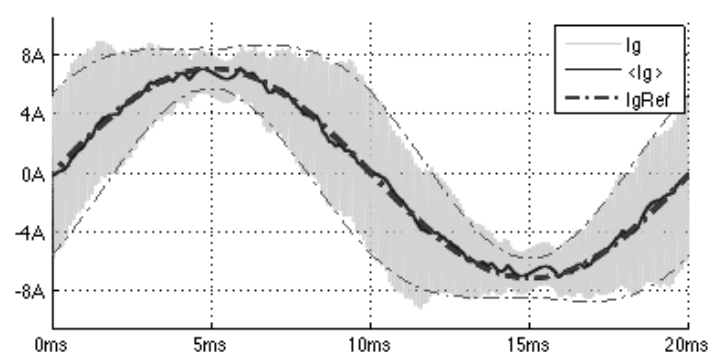

Figure 10. Closed-loop response of proposed controller

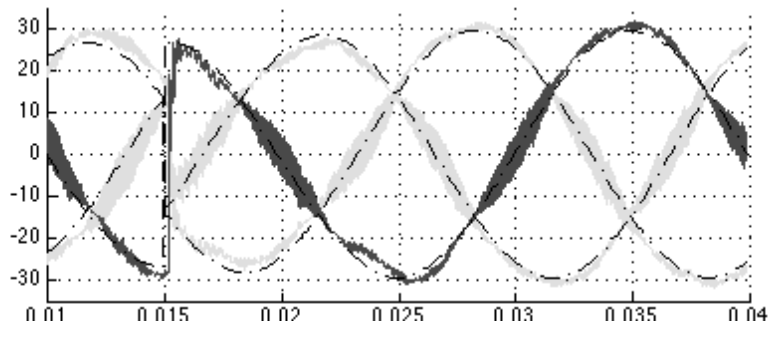

Figure 11. Line currents in transient from $+14 \mathrm{~kW}$ to $-14 \mathrm{~kW}$

\section{CONCLUSiOnS}

It is demonstrated that in grid-tied-inverter control, resonant integrators can be moved from the current loop to the input voltage filter allowing for better accuracy of voltage rms and frequency measurements. The design of the input filter is discussed to ensure good performance and phase compensation and not compromising controlability.

A robust feed-forward is built upon this filter that, together with the elimination of output distortion, results in a fair openloop control response. By closing the loop with a PI controller and by turning off supposedly non-conducting IGBTs, a current transient results that competes with state-of-the-art control strategies and is only limited by output filter inductance.

The filter is also dimentioned for fast critically dumped convergence, assuring the stability of frequency or voltage based power droop control that may be implemented on top of the current loop, and whose interaction with this controller should be aim of future study.

\section{REFERENCES}

[1] C. Busada and S. Jorge, "Current controller based on reduced order generalized integrators for distributed generation systems," IEEE Transactions on Industrial Electronics, vol. 59, no. 7, pp. 2898-2909, 2012.

[2] P. Rodriguez, A. Luna, I. Candela, R. Teodorescu, and F. Blaabjerg, "Grid synchronization of power converters using multiple second order generalized integrators," Industrial Electronics, 2008. IECON 2008. 34th Annual Conference of IEEE, pp. 755-760, 2008.

[3] O. Carranza, E. Figueres, G. Garcera, C. L. Trujillo, and D. Velasco, "Comparative study of Speed Estimators Applied to Wind Turbine with Harmonic Distortion in both the Currents and the Voltages," International Conference on Renewable Energies and Power Quality (ICREPQ), 2010.

[4] Z. Luo, M. Kaye, C. Diduch, and L. Chang, "Frequency measurement using a frequency locked loop," IEEE Energy Conversion Congress and Exposition (ECCE), pp. 917-921, Sep. 2011.

[5] K. Brabandere and T. Loix, "Design and operation of a phase-locked loop with Kalman estimator-based filter for single-phase applications," IEEE Industrial Electronics, (IECON) - 32nd Annual Conference on, pp. 525-530, 2006.

[6] M. Reza, M. Ciobotaru, and V. Agelidis, "Frequency adaptive linear Kalman filter for fast and accurate estimation of grid voltage parameters," Power System Technology (POWERCON), 2012 IEEE International Conference on, 2012.

[7] G. Grandi, J. . Loncarski, and R. Seebacher, "Effects of current ripple on dead-time distortion in three-phase voltage source inverters," Energy Conference and Exhibition (ENERGYCON), 2012 IEEE International, pp. 207-212, 2012.

[8] L. Ben-Brahim, "The analysis and compensation of dead-time effects in three phase PWM inverters,' Industrial Electronics Society (IECON) '98. Proceedings of the 24th Annual Conference of the IEEE, vol. 2, pp. 792$797,1998$. 